\title{
Automated mechanical chest compression as a bridge to primary percutaneous coronary intervention: Case report
}

\author{
Bebiana Faria, Marina Fernandes, Sílvia Ribeiro, António Lourenço \\ Hospital Senhora da Oliveira, Guimarães, Portugal
}

\begin{abstract}
In a peripheral hospital without primary percutaneous coronary intervention or mechanical circulatory support, therapeutic options in patients with myocardial infarction complicated with cardiogenic shock or refractory cardiopulmonary arrest are very limited.

We report a case of a 59-year-old man with acute myocardial infarction with ST-segment elevation complicated by cardiogenic shock and refractory cardiac arrest, transported with ongoing fibrinolysis to another hospital for rescue percutaneous coronary intervention in cardiopulmonary resuscitation with automated mechanical chest compression. This case underlines the difficulty in managing these critically ill patients in peripheral hospitals without advanced resources and discusses some therapeutic options, highlighting automated mechanical chest compression as a bridge to a percutaneous revascularization procedure.
\end{abstract}

\section{Introduction}

Acute coronary syndrome is the most common cause of Cardiogenic Shock (CS), mainly through a large zone of myocardial ischemia or a mechanical complication. ${ }^{1}$ CS complicates $6-$ $10 \%$ of all acute myocardial infarction with ST-segment elevation (STEMI) and remains a leading cause of death, with in-hospital mortality rates of $50 \% .^{2,3}$ In a peripheral hospital without hemodynamic laboratory or mechanical circulatory support, therapeutic

Correspondence: Bebiana Manuela Monteiro Faria, Rua dos Cutileiros 4835-044 Guimarães

Tel.: +351934899908

E-mail: bebianafaria@gmail.com

Key words: Acute coronary syndrome; cardiogenic shock; cardiac arrest; automated mechanical chest compression.

Conflict of interest: the authors declare no potential conflict of interests.

Ethics approval and consent to participate: The manuscript does not contain any elements that would allow the recognition of the patients.

Received for publication: 21 October 2019.

Accepted for publication: 18 May 2020

This work is licensed under a Creative Commons Attribution 4.0 License (by-nc 4.0).

(C) Copyright: the Author(s), 2020

Licensee PAGEPress, Italy

Emergency Care Journal 2020; 16:8636

doi:10.4081/ecj.2020.8636 options in patients with myocardial infarction complicated with CS or refractory Cardiorespiratory Arrest (CA) are very limited and not consensual.

\section{Case Report}

A 59-year-old man with a personal history of hypertension, obesity, smoking and epilepsy, was admitted to the emergency department of a peripheral hospital (without primary Percutaneous Coronary Intervention (PCI) or mechanical circulatory support capacity) due to oppressive chest pain, breathlessness, associated with an altered state of consciousness, within an hour of symptoms onset.

Physical examination showed non-measurable blood pressure, symmetrical filiform peripheral pulses with a regular frequency of $100 \mathrm{ppm}$, signs of peripheral poor perfusion, Glasgow Coma Scale of 10 (E3V2M5), cardiac auscultation without murmurs and pulmonary auscultation with diffuse crackles.

A 12-lead electrocardiogram (Figure 1) revealed sinus rhythm with a heart rate of $106 \mathrm{bpm}$, with diffuse ST-segment elevation, more evident in the anterolateral wall. Transthoracic echocardiogram showed severely left ventricular dysfunction with contraction of the basal segment of the interventricular septum and remaining segments akinetic, without evidence of pericardial effusion, aortic flap or mechanical complications.

Towards these findings, the diagnosis of STEMI complicated by CS was considered. The patient received anti-thrombotic loading doses, initiated vasopressor with noradrenaline at $1 \mathrm{mcg} / \mathrm{kg} / \mathrm{min}$ and invasive ventilatory support.

While preparing the patient for transfer to the hospital with primary PCI capability, CA was observed in Pulseless Electrical Activity (PEA). Advanced Life Support (ALS) was immediately initiated. Four cycles were performed with no Recovery Of Spontaneous Circulation (ROSC). At this time, it was infused fibrinolysis with tenecteplase. ALS was maintained for about $20 \mathrm{~min}-$ utes with no signs of ROSC, always in PEA. It was placed an Automated Mechanical Chest Compression device (AMCC) (LUCAS $2^{\circledR}$ ) and started transport to the hospital with primary PCI capability. During Cardiopulmonary Resuscitation (CPR) with manual compressions the maximum observed $\mathrm{ETCO}_{2}$ was $12 \mathrm{mmHg}$ and with AMCC it was $18 \mathrm{mmHg}$. After 20 minutes of transport, the patient was admitted at the hemodynamic laboratory in CPR under AMCC, with a total CPR time of 50 minutes and 40 minutes after fibrinolysis.

Coronary angiography showed thrombotic occlusion of the left main (Figure 2), and angioplasty was performed by stent implantation from the left main to the left anterior descending coronary artery (Figure 3). After angioplasty, spontaneous circulation returned. Echocardiogram revealed very severe left ventricular dysfunction and vasopressor support was restarted with increasing doses. Two hours after angioplasty, asystole was verified, and the patient died. 


\section{Discussion}

In STEMI patients presenting with CS in which PCI is estimated to occur $120 \mathrm{~min}$ after diagnosis, immediate fibrinolysis and transfer to a PCI center should be considered. In these cases, as soon as arrive at the PCI center, emergent angiography is indicated, regardless of the ST resolution and the time from fibrinolysis administration. ${ }^{2}$

Fibrinolytic treatment in the context of refractory CA in STEMI is not well established and showed no improvement in survival at hospital discharge, ${ }^{3,4}$ however, in this case it was administered, although off-label, since the diagnosis of STEMI was performed after witnessed and promptly assisted CA.

At best, standard manual CPR produces coronary and cerebral perfusion that is just $30 \%$ of normal. Providing high-quality manual chest compressions can be challenging and there is evidence that CPR quality deteriorates with time. Although there are no differences in clinical outcomes compared manual CPR to AMCC devices, AMCC may enable the delivery of high-quality compressions especially in difficult circumstances: patients' transporting in an ambulance to receive complex treatment, prolonged CPR (e.g. during fibrinolytic therapy) and CPR during certain procedures (e.g. coronary angiography), many of these characteristics verified in this case. ., $^{5}$

Extracorporeal CPR (eCPR) with veno-arterial extracorporeal membrane oxygenation should be considered as rescue therapy for those patients in whom initial ALS measures are unsuccessful and/or to facilitate specific interventions like PCI. Observational studies suggest eCPR for CA is associated with improved survival when there is a reversible cause for cardiac arrest (e.g. myocardial infarction). ${ }^{5}$ Probably eCPR would have been a good strategy for this patient, however, he was admitted to a hospital without mechanical circulatory support.

There is limited evidence for recommending routine transport to a hospital with ongoing CPR in case of CA during STEMI. The decision will depend on patient selection and must take into account a realistic chance of survival, witnessed CA with an initial shockable rhythm, bystander CPR and intermittent signs of ROSC. There are no large outcome studies available, but small case series suggest benefit in these selected cases. ${ }^{7}$

In this clinical case, CA was witnessed and immediately assisted, fibrinolytic treatment was started after CA, and despite being a non-defibrillate rhythm, the patient was young, within an hour of symptom onset. While facing a refractory CA, under fibrinolytic

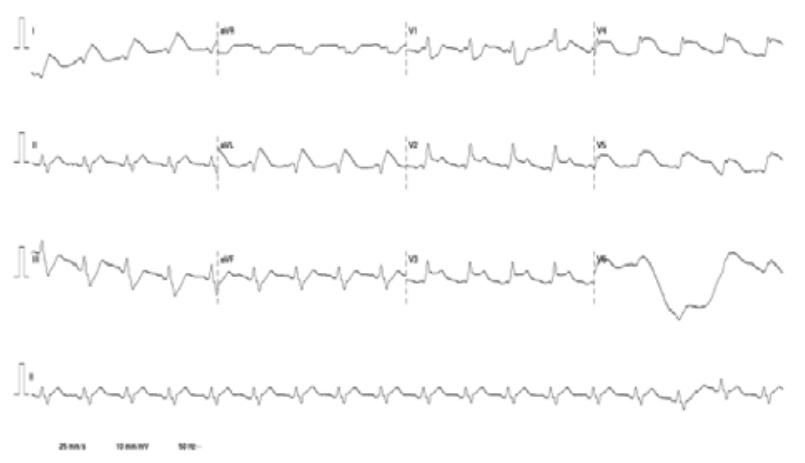

Figure 1. Admission 12-lead electrocardiogram revealed sinus rhythm with a heart rate of $106 \mathrm{bpm}$, with diffuse ST-segment elevation, more marked in the anterolateral wall. treatment in a peripheral hospital, with a hemodynamic lab at 20 minutes distance, and it was possible to ensure high-quality cardiopulmonary resuscitation with the AMCC, it was decided to transport the patient in CPR to rescue PCI. After left main angioplasty spontaneous circulation was observed, however, a few hours later the patient died. In this case, the cardiac arrest would probably have been more secondary to pump failure than ischemia, given the rhythm of arrest. Possibly the outcome could be different if a defibrillate rhythm was presented.

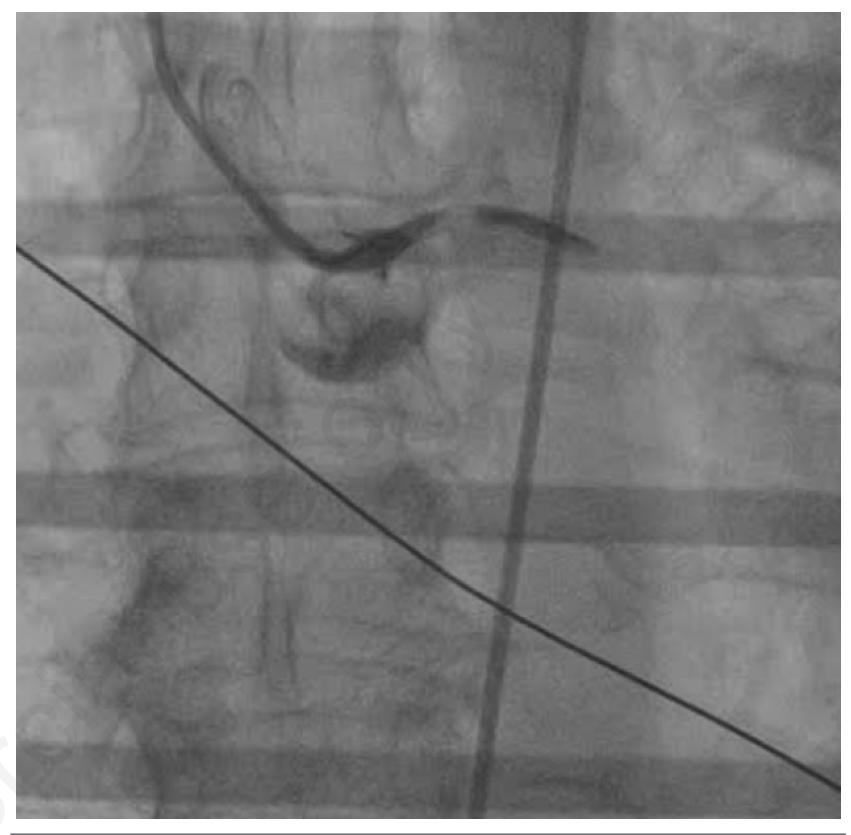

Figure 2. Coronary angiography showing thrombotic occlusion of the left main.

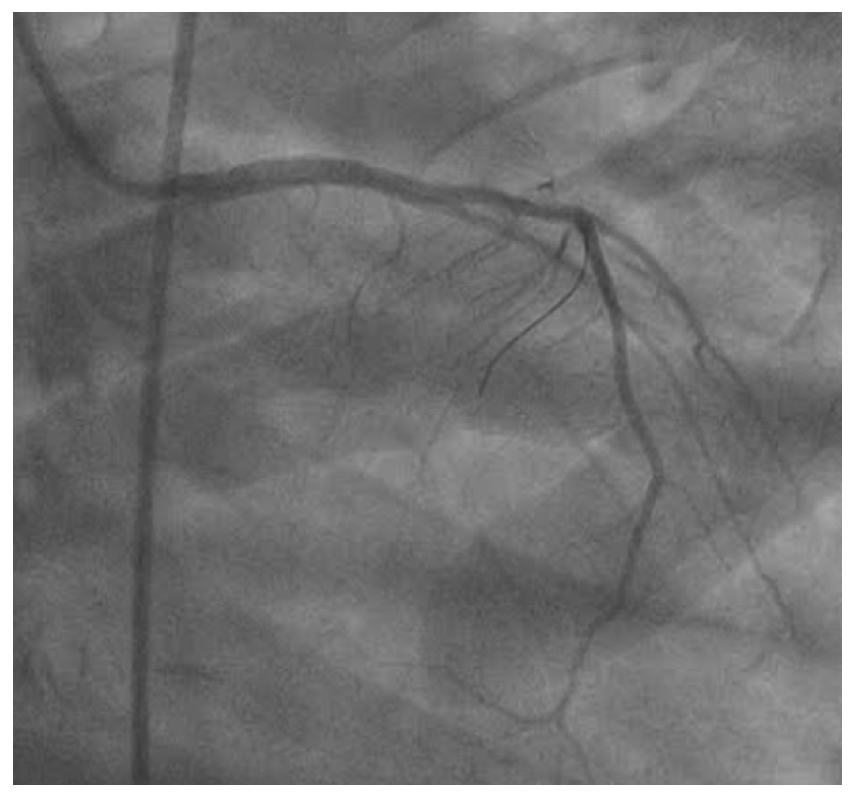

Figure 3. Coronary angiography after angioplasty with stent implantation from the left main to the anterior descending artery. 


\section{Conclusion}

Transport of the patient to primary angioplasty in CPR is not consensual, there are no controlled studies confirming the outcome, however, reports of small series suggest that there may be a benefit in selected cases, especially, when CA is witnessed and high-quality cardiopulmonary resuscitation is possible. The AMCC allows ensuring high-quality CPR, especially in long resuscitation. In this case, the AMCC enabled coronary revascularization and consequently the return of spontaneous circulation. However, the degree of left ventricular dysfunction, more than ischemia, determined the prognosis of the patient.

\section{References}

1. Nikolaoua N, Arntz HR, Bellou A, et al. European Resuscitation Council Guidelines for Resuscitation 2015 Section 8. Initial management of acute coronary syndromes. Resuscitation 2015; 264-77.

2. Ibanez B, James S, Agewall S, et al. 2017 ESC Guidelines for the management of acute myocardial infarction in patients presenting with ST-segment elevation. Eur Heart J 2018;39:11977.

3. Bernoche C, Timerman S, Polastri T et al. Atualização da Diretriz de Ressuscitação Cardiopulmonar e Cuidados Cardiovasculares de Emergência da Sociedade Brasileira de Cardiologia - 2019. Arq Bras Cardiol 2019;113:449-663.

4. Böttiger BW, Arntz HR, Chamberlain DA, Bluhmki E, Belmans A, Danays T, et al. Thrombolysis during resuscitation for out-of-hospital cardiac arrest. $N$ Engl J Med 2008;359:2651-62.

5. Soar J, Nolan J, Böttiger B, et al. European Resuscitation Council Guidelines for Resuscitation 2015 Section 3. Adult advanced life support. Resuscitation 2015;100-47.

6. Lafuente-Lafuente C, Melero-Bascones M. Active chest compression-decompression for cardiopulmonary resuscitation. Cochrane Database Syst Rev 2013;2013:CD002751.

7. Truhlár A, Deakinc C, Soar J, et al. European Resuscitation Council Guidelines for Resuscitation 2015 Section 4. Cardiac arrest in special circumstances. Resuscitation 2015;148-201. 